PAPER

\title{
Spatial backward planar projection in absorbing media possessing an arbitrary dispersion relation
}

\author{
Gregory T. Clement* \\ Department of Radiology, Harvard Medical School, Brigham and Women's Hospital, \\ 221 Longwood Ave RM 521, Boston, MA 02115 USA
}

(Received 25 September 2009, Accepted for publication 1 May 2010)

\begin{abstract}
Planar projection methods have been shown to rapidly relate fields between two planes. Such an approach is particularly useful for characterizing transducers, since only a single plane needs to be measured in order to characterize an entire field. The present work considers the same approach in the presence of an arbitrary dispersion relation. Unlike traditional methods that use Fourier solutions of the time-domain wave equation, the approach starts from a frequency-domain Helmholtz equation for waves in a dispersive medium. It is shown that a transfer function similar to that derived from time domain equations can be utilized. Both the forward- and backward-projection behaviors are examined and it is demonstrated that the approach is invariant to propagation direction.
\end{abstract}

Keywords: Planar projection, k-space, Dispersion, Ultrasound

PACS number: 43.35.Bf, 43.35.Yb, 43.80.Vj [doi:10.1250/ast.31.379]

\section{INTRODUCTION}

A variety of linear planar projection algorithms in the wavevector-frequency domain [1-3] and the wavevectortime domain [4-6] have been described for rapidly computing acoustic waves between positions in space/ time. Generally, these methods are derived from Fourier solutions to the time-domain linear wave equation. Such an approach, however, limits the dispersion to a fixed relation characterized by the particular wave equation. Thus, anomalous dispersion in biological tissue and other materials can only be approximated by such specific equations.

A more general time domain wave equation for materials that follow a frequency power law was expressed by Szabo [7], in terms of the linear lossless wave equation and a convolution loss operator, which has subsequently been modified and used to describe a variety of situations in the time domain, assuming a power law dependence. Moreover, Waters et al. [8] showed this representation could be extended to general distributions. Variations in the approach center on efficient methods to model the timedomain solutions [9-12].

On the other hand, the general case of anomalous dispersion can be handled with relative ease in wave-vector space [13]. The present work indicates how the frequency-

\footnotetext{
*e-mail: gclement@hms.harvard.edu
}

domain wave equation can be further transformed into the wavevector-frequency domain, where it has a known solution. It will be shown that this solution can be used for planar projection and is valid under arbitrary dispersion conditions. Moreover, the selection of a specific dispersion relation readily relates the equation to the most commonly used time-domain equations.

A key aspect of planar projection is its ability to backpropagate a signal toward its source. It is demonstrated that the projection property is still valid in dispersive media. Utility of such projections may include a wide range of applications where the angular spectral method is applied, including transducer field characterization in lossy media, the prediction of fields through homogeneous and layered media, and reconstruction of fields via back-projection.

\section{THEORY}

\subsection{The Generalized Dispersive Wave Equation}

The present linearized theory is based on a dynamic equation of state, which leads to the wave equation in dispersive media [14],

$$
\nabla^{2} P(\boldsymbol{r}, \omega)+\frac{\omega^{2}}{C(\omega)^{2}} P(\boldsymbol{r}, \omega)=0,
$$

where $P$ is the Fourier transform of pressure with respect to time and $C$ is the complex sound speed. Frequency dependence on $C$ prevents straightforward expression of (1) in the time domain, thus motivating frequency domain 
modeling [15]. However, rather than propagate the wave purely in space, (1) can be transformed with respect to the Cartesian orthogonal coordinates, $x$ and $y$ such that the equation takes the form of the ordinary differential equation

$$
\frac{\partial}{\partial z} \tilde{P}\left(k_{x}, k_{y}, z, \omega\right)+K^{2} \tilde{P}\left(k_{x}, k_{y}, z, \omega\right)=0,
$$

where

$$
K^{2}=\frac{\omega^{2}}{C^{2}}-k_{x}{ }^{2}-k_{y}{ }^{2},
$$

and $\tilde{P}$ is the Fourier transform of pressure with respect to the Cartesian $x$ and $y$ dimensions, and time.

The equation is identical in form to that used in the angular spectrum approach, with the important distinction that $C$ is an arbitrary complex function of frequency. The relevant known solution to (2) in terms of the initial pressure is given by

$$
\tilde{P}=\tilde{P}_{0} e^{i K\left(z-z_{0}\right)} .
$$

\subsection{Relation to Lossy Time Domain Equations}

Before considering the case of an anomalous dispersion, it is instructive to illustrate how (2) and (3) readily reduce to common linear equations in the time domain by defining $C$. It may be readily verified that setting $C=c_{0}$, where $c_{0}$ is a real constant, gives the transformed form of the standard lossless equation,

$$
\nabla^{2} p(\boldsymbol{r}, t)-\frac{1}{c_{0}^{2}} \frac{\partial^{2}}{\partial t^{2}} p(\boldsymbol{r}, t)=0 .
$$

Substitution of the value $C=c_{0} / \sqrt{1+i \omega \delta / c_{0}^{2}}$ into Eq. (2) and Fourier transformation yields a linearized form of the Westervelt equation $[16,17]$,

$$
\nabla^{2} p(\boldsymbol{r}, t)-\frac{1}{c_{0}^{2}} \frac{\partial^{2}}{\partial t^{2}} p(\boldsymbol{r}, t)+\frac{\delta}{c_{0}} \frac{\partial^{3}}{\partial t^{3}} p(\boldsymbol{r}, t)=0 .
$$

Similarly setting $C=c_{0} \sqrt{1+i \tau \omega}$ gives the transformed form of the linearized Stokes equation [18], whose dispersion relation was previously described [19] for forward planar projection,

$$
\left(1+\tau \frac{\partial}{\partial t}\right) \nabla^{2} p(\boldsymbol{r}, t)-\frac{1}{c_{0}^{2}} \frac{\partial^{2}}{\partial t^{2}} p(\boldsymbol{r}, t)=0 .
$$

The relaxation time for the medium is given by $\tau$.

Finally, setting $C=c_{0} / \sqrt{1+2 i c_{0} \alpha|\omega|^{y} / \omega}$, the generalized power loss equation expressed by Szabo [7] remains in its manageable frequency domain form, with $y$ a real number that gives the power relation. This equation was derived by induction in Szabo's work for describing lossy media of the power law type. This behavior concerns a large range of physical problems in underwater acoustics and medical ultrasound where loss is dependent on frequency [7]. When $C$ is substituted into (2) the Fourier transform is not trivial, unless $y$ is an even integer. However, through the use of generalized functions, it may be shown that in the space-time domain the equation becomes:

$$
\begin{aligned}
& \nabla^{2} p(\boldsymbol{r}, t)-\frac{1}{c_{0}^{2}} \frac{\partial^{2}}{\partial t^{2}} p(\boldsymbol{r}, t)+L_{\gamma} \otimes p(\boldsymbol{r}, t)=0 \\
& L_{\gamma}=\frac{2}{c_{0}} \frac{-\alpha_{0} \Gamma(y+2) \cos \left[\frac{\pi(y+1)}{2}\right]}{\pi|t|^{y+2}}
\end{aligned}
$$

where $\Gamma$ represents the gamma function and $\otimes$ defines a convolution operator. For detailed derivation leading to Eq. (8), the reader is directed to Szabo's original paper. Comparison between Eqs. (8) and (4) indicate a significant difference in complexity between spatial planar projection and the temporal representation of the same equation.

\subsection{Backward Projection}

One advantage of the approach is the ability to propagate both toward and away from the source [20]. Thus, a signal can be recorded away from the source, and then back-projected to give information about the signal near a transducer face, assuming the propagating field is contained within the measurement plane, $z_{0}$. This ability can be contrasted with time reversal [21], which is violated in the presence of the absorption term.

As Hallaj et al. [22] have noted, given that $p(\boldsymbol{r}, t)$ is a solution to the time domain equation, a general condition for time-reversal invariance is that $p(\boldsymbol{r},-t)$ is also a solution. By the time reversal property of the Fourier transform,

$$
p(\boldsymbol{r},-t) \stackrel{F T}{\Leftrightarrow} P(\boldsymbol{r},-\omega),
$$

it can be seen from (1) that a necessary and sufficient condition for invariance is

$$
C(\omega)=C(-\omega) .
$$

Now, in analogy with time invariance, if $\tilde{P}\left(k_{x}, k_{y}, z, \omega\right)$ is a solution to (2), validity of backward projection requires that $\tilde{P}\left(k_{x}, k_{y},-z, \omega\right)$ is also a solution. By direct substitution, it may readily be verified that this is the case. Thus, (2) is invariant with respect to the spatial dimension.

\section{NUMERIC EXAMPLE}

To illustrate the approach, signals radiating from a 30-mm diameter circular piston radiator were considered. A Gaussian-shaded temporal pulse was radiated from the piston face, which was situated at the origin of a Cartesian axis, in the plane normal to the $z$-axis, as illustrated in Fig. 1. A center temporal frequency of $1 \mathrm{MHz}$ and $-3 \mathrm{~dB}$ bandwidth of $251 \mathrm{KHz}$ was used for all simulations. The 
a.

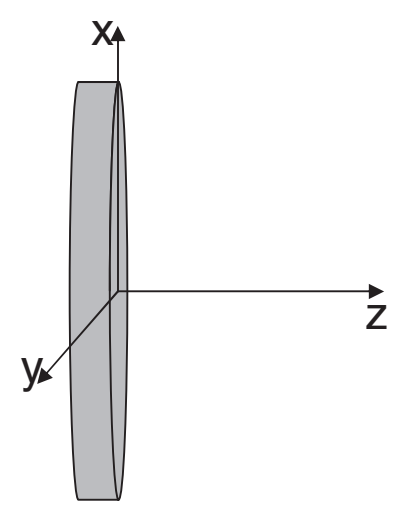

b.

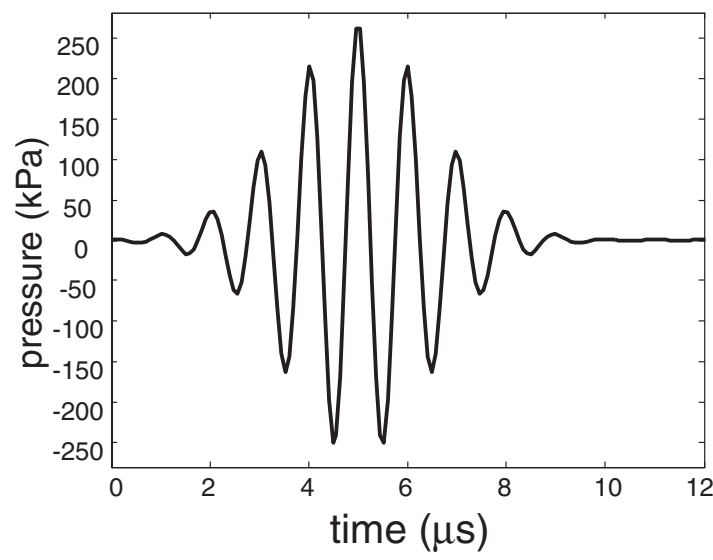

C.

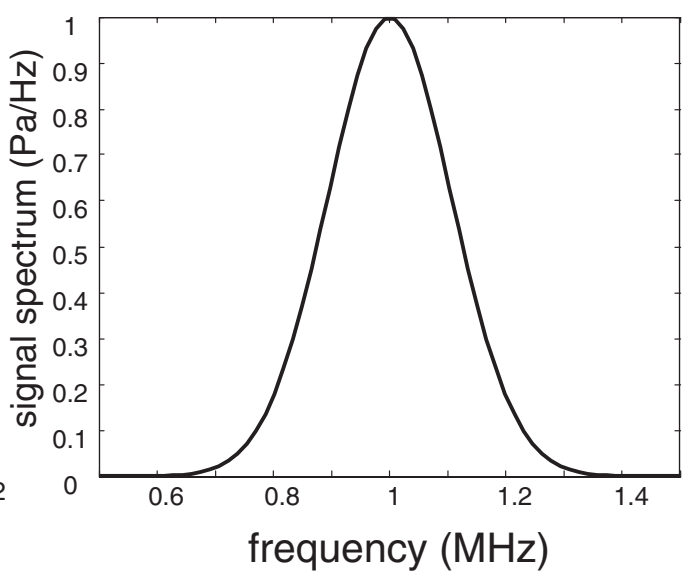

Fig. 1 (a) The geometry of the projection problem, with a circular planar source, (b) the initial time-trace of the signal across the source, and (c) The Fourier transform of the signal.

a.

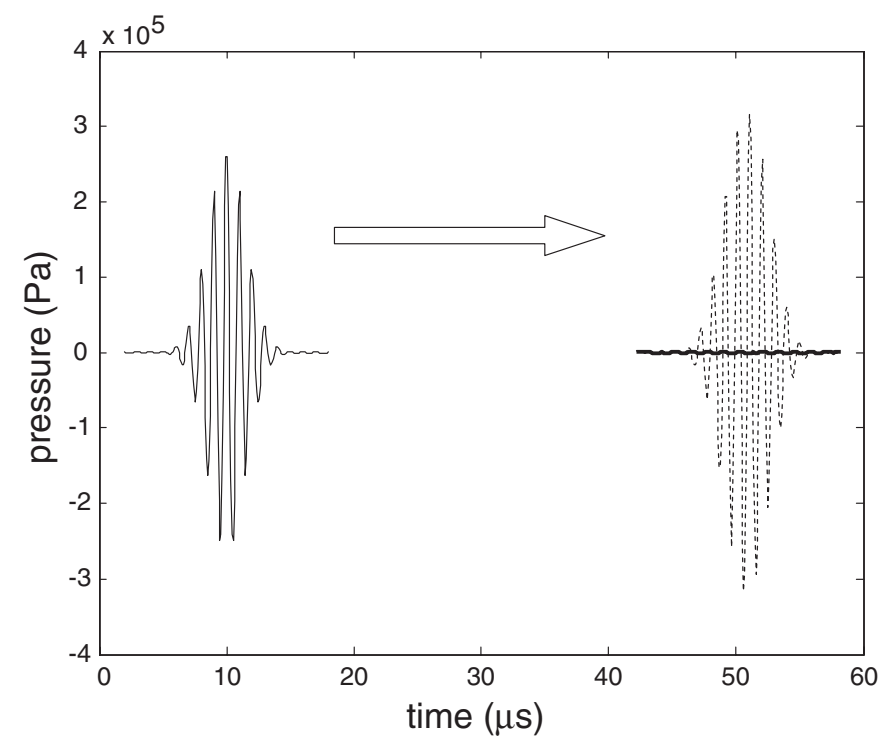

b.

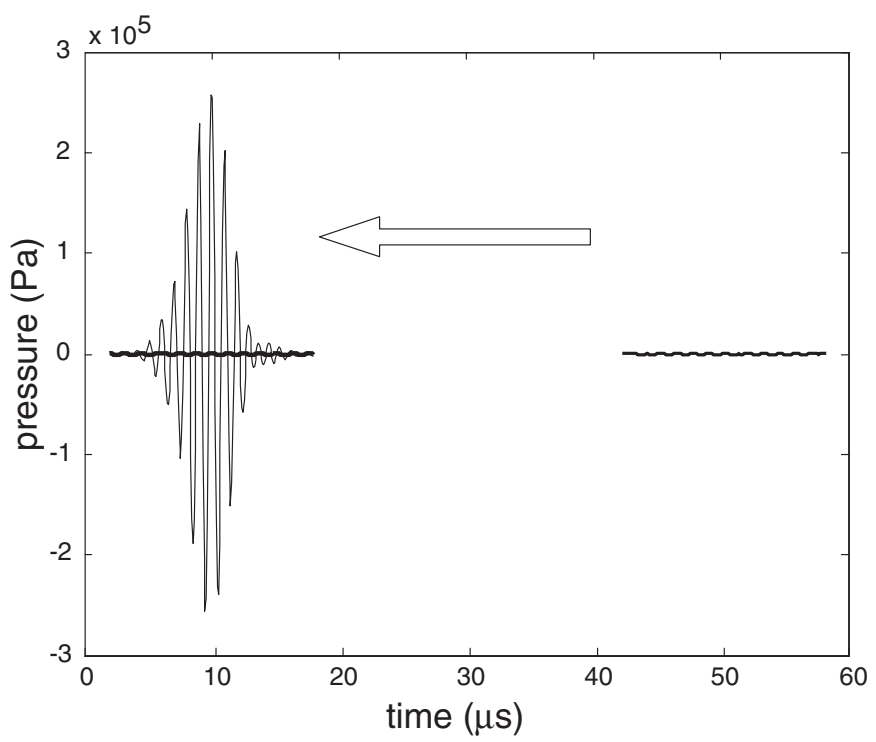

Fig. 2 (a) The initial signal at $z=0 \mathrm{~mm}$ (left, solid) is projected forward to $z=60 \mathrm{~mm}$ in an ideal medium (dashed), and in a lossy, dispersive medium (solid). (b) The lossy signal (right) at $z=60 \mathrm{~mm}$ is then used as a source and back-projected to $z=0 \mathrm{~mm}$ under ideal conditions (smaller signal, left), and with absorption and dispersion (larger signal, left).

simulation input was a 3D dataset, $P\left(x, y, z_{0}, \omega\right)$, representing the signal frequency content over the $x-y$ plane at $z=0$. Initial values were expressed as an input grid of $100 \times$ $100 \times 64$ points, representing the two spatial dimensions and the frequency, respectively. The spatial resolution was $0.3 \mathrm{~mm}$ in both spatial dimensions and the frequency resolution was $0.016 \mathrm{MHz}$.

A low pass spatial filter was added in order to eliminate explosion from exponentially increasing round-off error during back-projection. That is, higher spatial frequencies are known to lead to non-propagating evanescent wave solutions when $k_{x}{ }^{2}+k_{y}{ }^{2}>\operatorname{Re}\left\{\frac{\omega^{2}}{C^{2}}\right\}$. The cutoff was set at $0.7 \operatorname{Re}\left\{\frac{\omega^{2}}{C^{2}}\right\}$, removing all evanescent waves as well as the upper $30 \%$ of the higher spatial frequencies which, due to the directivity of the beam, were not expected to significantly affect the results. Specifically, $P\left(k_{x}, k_{y}, z=\right.$ $0 \mathrm{~mm}, \omega)$ above the cutoff frequency contained a mean value equal to $0.3 \%$ of the peak found at $P(0,0, z=$ $0 \mathrm{~mm}, \omega)$, with a maximum value equal to $1.3 \%$ of this peak.

Details of the algorithm follow a previous description by Clement [19], but with modification allowing for an arbitrary dispersion relation. In the former work, the algorithm was experimentally validated for the case of forward projection through absorbing layers. The present version of the algorithm was implemented using Matlab, on 

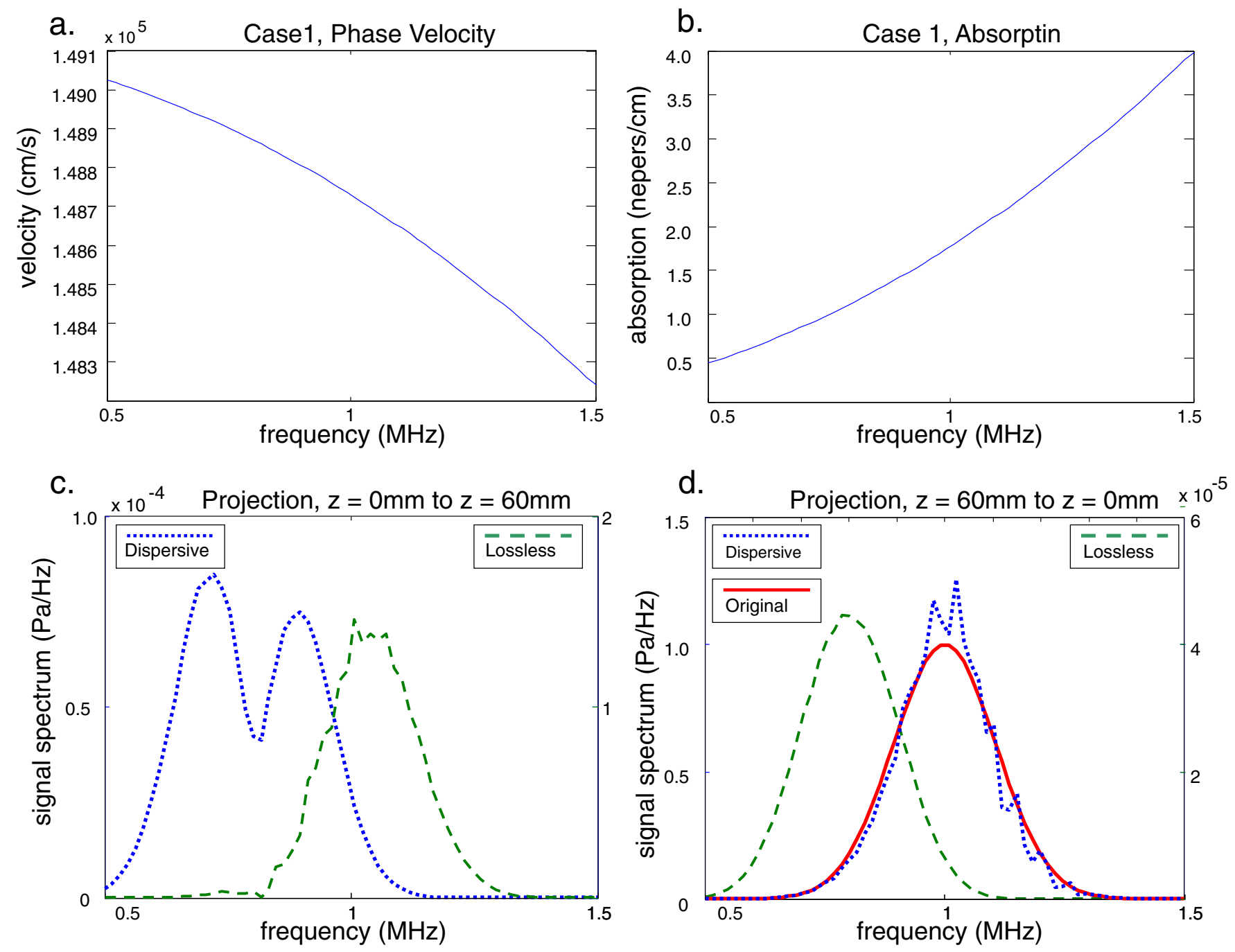

Fig. 3 (a) The real part of the sound speed and (b) the absorption coefficient used in the first simulation. (c) The frequency content at $z=60 \mathrm{~mm}$ is reduced in the dispersive case (dotted), as compared to the ideal case (dashed). (d) Frequency content of the back-projection both neglecting dispersion (dashed) and taking into account dispersion (dotted) is compared to the initial signal (solid).

a XP 64-bit operating system. The hardware consisted of two dual-core $3 \mathrm{GHz}$ Xeon processors, and $8 \mathrm{~GB}$ of RAM. CPU time was monitored over the entire simulation. Typical processing times of $8 \mathrm{~s}-10 \mathrm{~s}$ were observed, with a memory requirement of approximately $1.25 \mathrm{~GB}$.

Simulated signals were propagated through a dispersive medium and "measured" over the $x-y$ plane at a distance $z_{0}$ away from the source. The propagated signal was compared in both the frequency domain and the time domain with a second signal that was calculated using $C=1,498 \mathrm{~m} / \mathrm{s}$. Figure 2(a) illustrates a projection from $z=0 \mathrm{~mm}$ to $z=60 \mathrm{~mm}$, which was performed in an ideal medium, and in a lossy, dispersive medium. The projected dispersive signal was then used as the source and backprojected to the position of the transducer face, where it was compared with the original signal (Fig. 2(b)). For this example, calculations were performed using a velocity distribution given in Fig. 3(a) and attenuation value as shown in 3(b). In this manner, the back-projection simulated the process of characterizing a transducer by measuring a field and then back-projecting it to the source location. For reference, the "measured" signal was also back-projected neglecting dispersion.

The dispersion relation described by the frequencydependent phase velocity given in Fig. 3(a) and attenuation given by 3(b) describe velocity [23] and absorption [24] distributions that are within the range that may be found, for example, in human cancellous bone. The field was projected forward to the plane $z=60 \mathrm{~mm}$ from the source. Figure 3(c) shows the attenuation and low pass filtering of the signal taken along the origin, $P(0,0, z=60 \mathrm{~mm}, \omega)$ as compared to the non-dispersive case $(C=1,491 \mathrm{~m} / \mathrm{s})$. The time history of the dispersive signal along the $x$-axis $(y=0, z=60 \mathrm{~mm})$ is provided for reference in Fig. 4.

The projected dataset was next used to simulate a field measurement acquired at $z=60 \mathrm{~mm}$, and back-projected to 


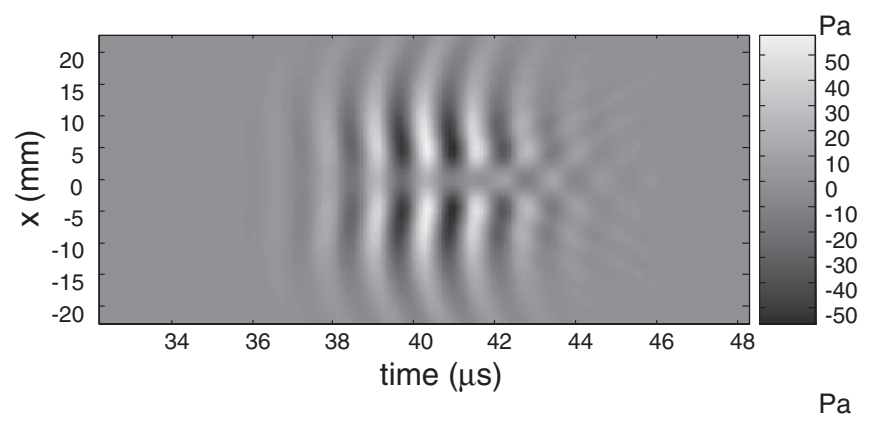

Fig. 4 The signal projected to $z=60 \mathrm{~mm}$.

$z=0 \mathrm{~mm}$ taking dispersion into account. For reference, a lossless back-projection was also performed using the same starting dataset. The frequency domain plot in Fig. 3(d) provides the spectrum on-axis after back-projection. The plot illustrates how the higher frequency components of the signal were reconstructed when dispersion was taken into account (left vertical axis). This spectrum may be contrasted with the lossless back-projection (right vertical axis), which reconstructs a spectrum lower in both peak frequency and amplitude than the actual starting signal. Differences in the spectra result in the time-domain differences given in Fig. 5, which shows the restoration of the signal phase and spatial localization.

A similar series of projections was carried out using tabulated data for the sound speed and absorption in human skull bone [25]. As shown in Figs. 6(a) and 6(b), the sound speed is approximately constant from 0.5 to $1.5 \mathrm{MHz}$, while the absorption increases more than a factor of 8 . The data were first projected from $z=0$ forward to $z=14 \mathrm{~mm}$, the assumed thickness of the bone. The projected datasets were then used as simulated field measurements and backprojected to the source. The dimension was chosen due to be representative of thick skull bone [26]. Discrepancy in the spectrum is apparent in the plot along the origin, $P(0,0, \omega)$, as shown in Figs. 6(c) and 6(d) (right vertical axes provide scale for lossless propagation). Such discrepancy demonstrates how an improper choice of equations could potentially lead to erroneous results in aberration correction methods that rely on the prediction of transcranial fields.

Further evaluation the method was conducted by calculating the RMS mean difference between the known initial signal at the source, $p_{0}(x, y, t)$, and the back projected signals $p(x, y, t)$ :

$$
R=\sqrt{\frac{\sum_{x, y, t}\left|p(x, y, t)-p_{0}(x, y, t)\right|^{2}}{\sum_{x, y, t}\left|p_{0}(x, y, t)\right|^{2}}} .
$$

To provide a more reasonable comparison, the signal neglecting dispersion was increased by an amplification a.

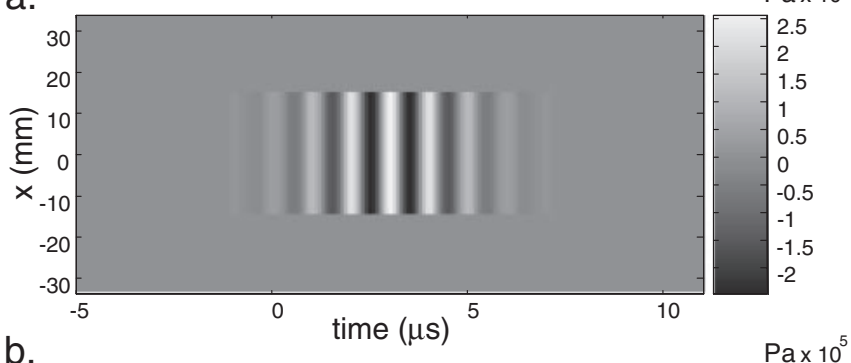

b.
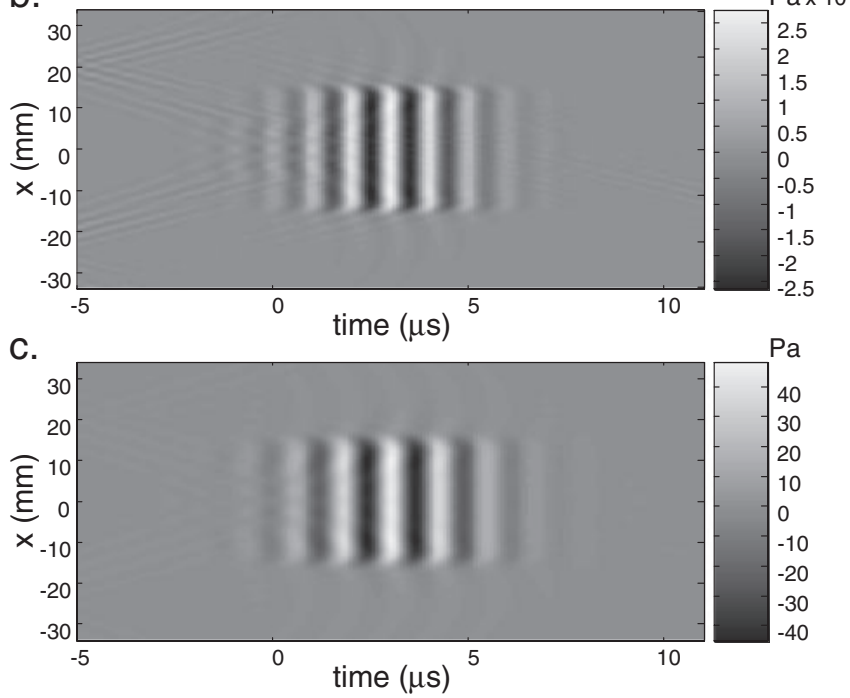

Fig. 5 The initial signal (a), is projected first to $z=$ $60 \mathrm{~mm}$, then back to the source (b). The same signal back-projected without consideration of dispersion (c).

factor, set so that the peak values of the initial and the projected waveforms (arbitrarily set at $0.268 \mathrm{MPa}$ ) were identical. In many applications, including time-reversal [22], it is common to offset attenuation by increasing initial signals by such factors. For the first numeric case shown in Fig. 3, an RMS difference of $19 \%$ was achieved for the dispersive signals, while the RMS difference without dispersion was $94 \%$. Both values may be compared to a baseline RMS difference of $1.6 \%$, obtained by projecting both forward and backward in a non-lossy medium, first to $z=60 \mathrm{~mm}$ then back to the source. This baseline provides an estimate on the accuracy of the technique under ideal conditions. A similar set of RMS difference measurement were made for the send case, presented in Fig. 6. An RMS difference of $16 \%$ was achieved for the dispersive signals, while the RMS difference without dispersion was $113 \%$.

The preceding examples were simulated using decidedly ideal conditions, the most notable being the absence of noise. Since noise could potentially have detrimental effects on back-projection, numeric projections were next performed in the presence of random noise. In this study, normally distributed random noise was generated using a pseudo-random generator and then added to the initial signal, which in the absence of noise was identical to the waveform in Fig. 4. The signal-to-noise ratio (SNR) was 

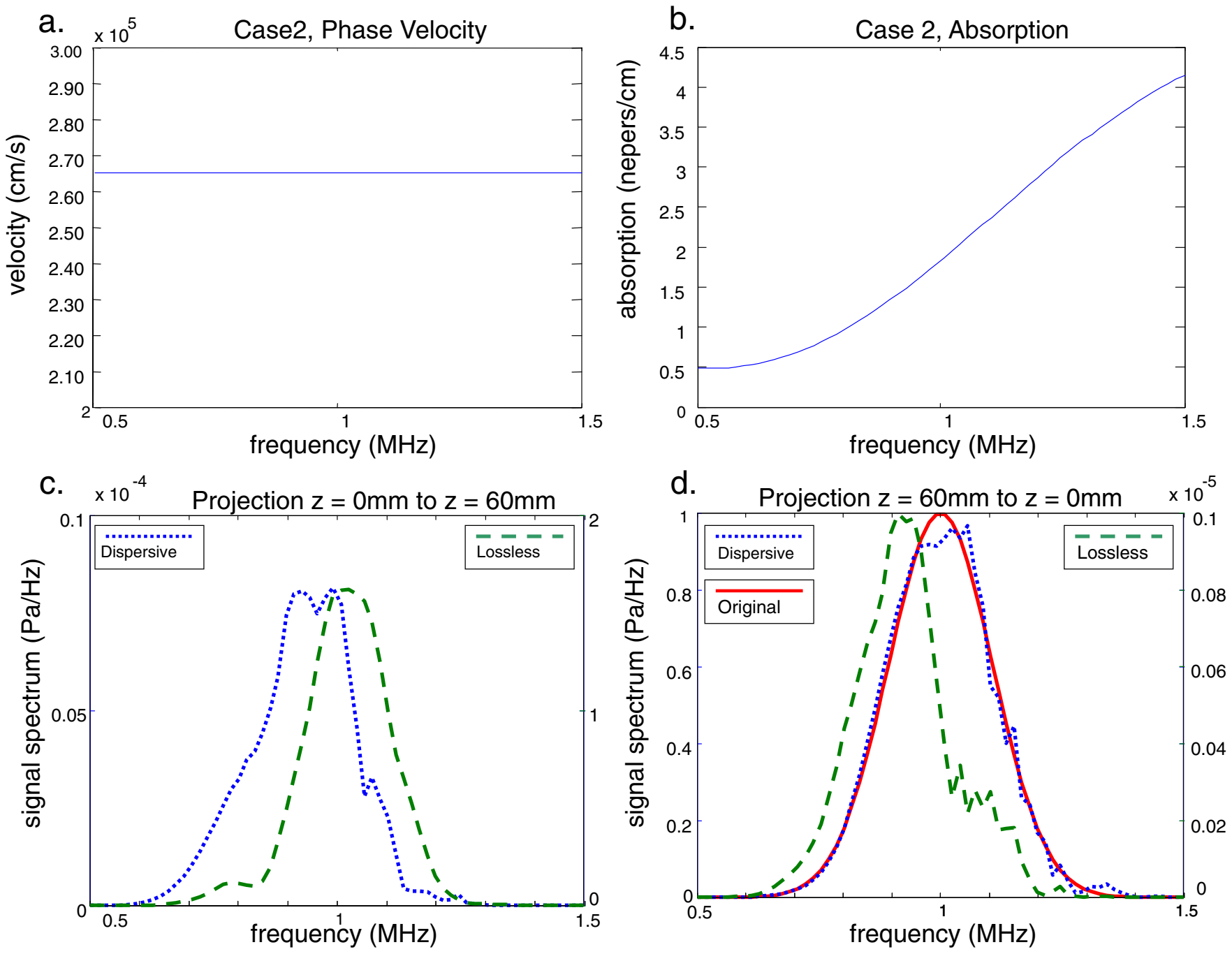

Fig. 6 (a) The real part of the sound speed and (b) the absorption coefficient used in the second simulation. (c) The frequency content at $z=14 \mathrm{~mm}$ (dotted), compared with the lossless case (dashed) for reference. (d) Frequency content of the back-projection both neglecting dispersion (dashed) and taking into account dispersion (dotted) is compared to the initial signal (solid).

determined by taking the ration between the initial waveform peak amplitude and the standard deviation of the data for all points away from the waveform. A range of SNR values between 0.1 and 40 was considered.

To regulate exponentially increasing error, a dynamic lowpass Butterworth filter was applied to the temporal frequency dimension, with a cutoff of approximately $1.22 \mathrm{MHz}$. Since the introduction of the filter itself can result in the loss of high-frequency signal components, simulation was first performed with the filter in place under idealized noiseless conditions. In this scenario, a modest increase in the RMS difference from 19\% (no filter) to $21 \%$ (temporal filtering) was observed. In the presence of noise, the algorithm was observed in all tested cases to be stable, provided that the bandpass filter was implemented. Figure 7(a) shows the on-axis initial pressure field before projection for SNR values of 2, 4 and 16, respectively. Figure 7(b) shows the corresponding fields after back-projection to the source. From the figure, it may be seen that although error grows with increasing noise, this error is distributed over the entire measurement volume so that the waveform is reconstructed even in a relatively noisy environment. A summery of the observed RMS difference as a function of SNR provided in Fig. 8, indicating decreased error with increasing signal that approaches the $21 \%$ error limit set by error in the method under idealized conditions.

\section{SUMMARY AND CONCLUSION}

This work described and demonstrated a numeric wavevector-frequency domain method for wave propagation that is valid for arbitrary dispersion relationships. As indicated in the work, limitations of the back-projection will ultimately be set by the signal-to-noise ratio (SNR). Nonetheless, significant improvement in the overall pressure fields were achieved by consideration of frequency 
a.
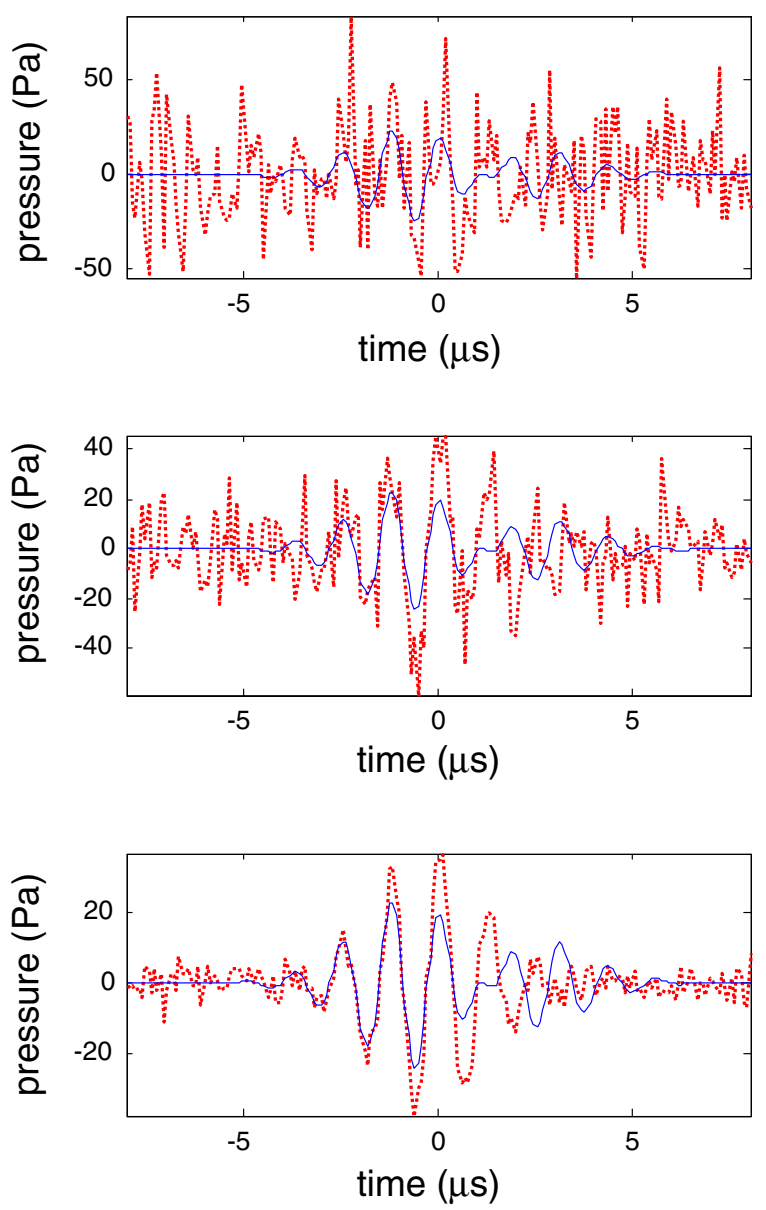

b.
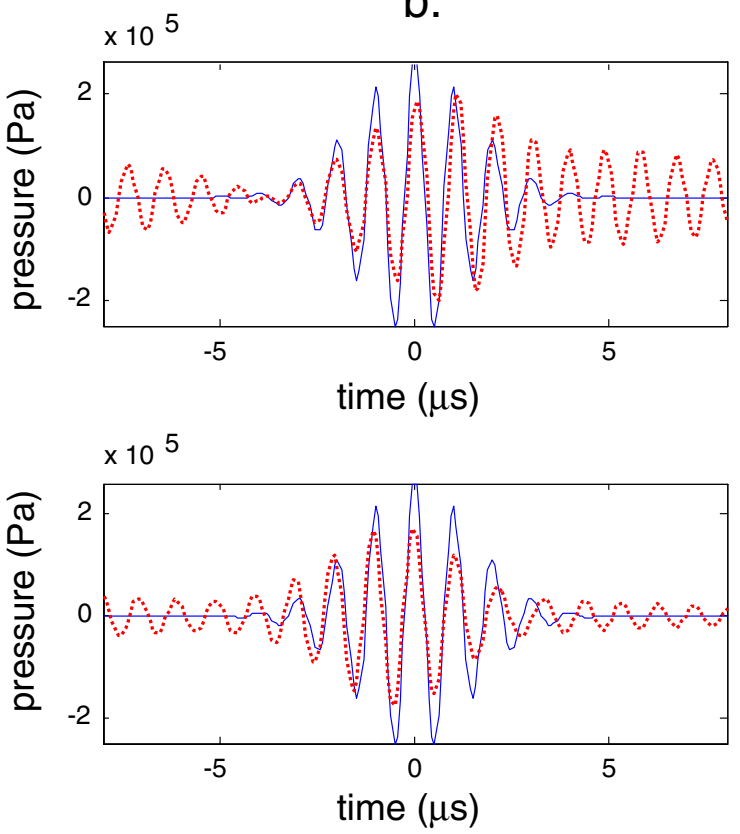

$\times 10^{5}$

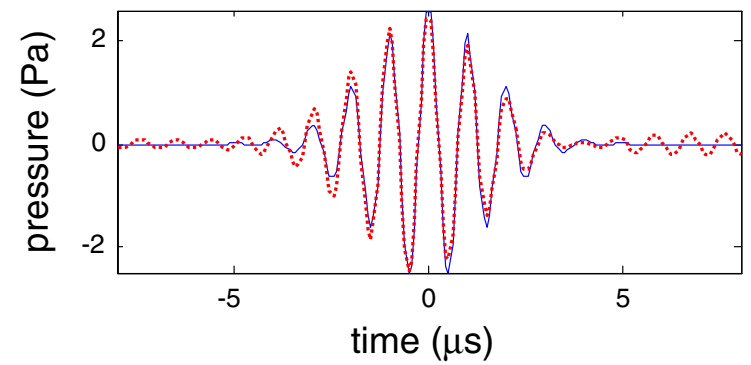

Fig. 7 (a) The on-axis acoustic pressures of the initial field at $z=60 \mathrm{~mm}$ to be back-projected (solid), and the same signals with the addition of gaussian noise (dotted). The rows represent SNR $=2$ (Top), SNR $=4$ (Middle), and SNR $=16$ (Bottom). (b) The fields corresponding to their counterparts in (a) after backward projection to $z=0$.

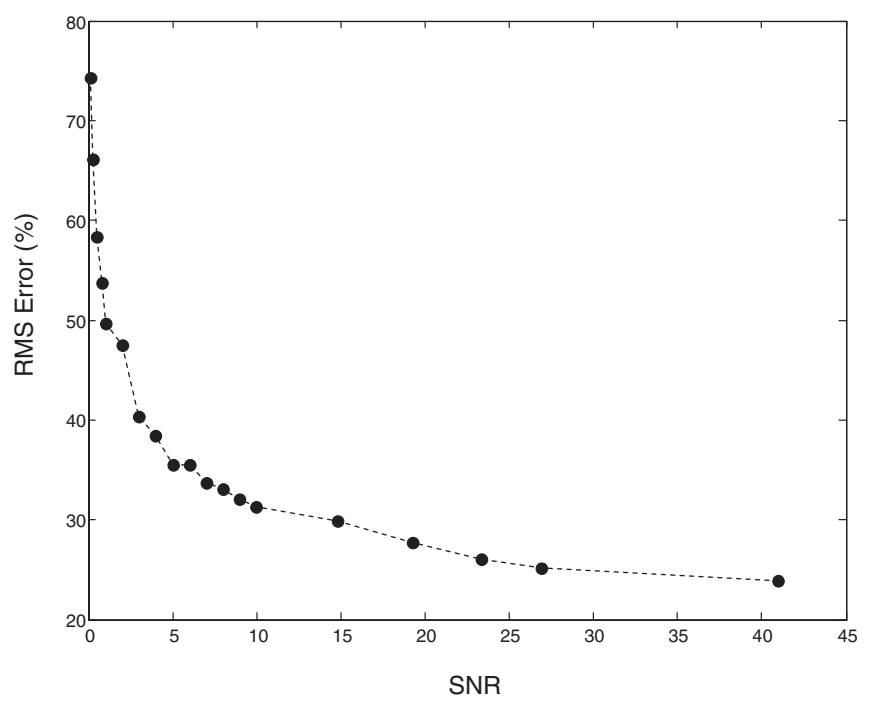

Fig. 8 Error as a function of Signal-to-Noise using the algorithm along with a temporal low pass filter. Error of the same data without noise was $21 \%$. dependent absorption and phase speed, without sacrifice to computation time or modeling complexity. It is stressed that the same measurement limitations are present even in lossless projection methods, due to diffractive spreading of the signal. Thus it is expected that in the dispersive case, like the lossless case, appreciable signal strength is required for accurate back projection, with the precise requisite SNR a function of the desired accuracy. Under the conditions of synthetic gaussian noise, the algorithm was found to be stable, provided that a low-pass temporal filter was added to the algorithm. In able to reconstruct the initial fields with correct frequency content, albeit with increased error.

Planar projection was validated under arbitrary dispersion conditions for both forward and backward propagation. This could prove particularly useful under conditions of anomalous dispersion, and provides a straightforward and computationally efficient method for predicting behavior in dispersive media. The current discussion was limited only to homogeneous situations, 
but the method is expected to be applicable under more general conditions [19].

While planar projection methods are known for their computational efficiency, their inherent operations in the frequency domain also make them ideal for operating dispersive media. Furthermore, symmetry of the spatial dimension allows back-propagation which remains invariant, regardless of temporal complexity.

\section{ACKNOWLEDGEMENTS}

This work was supported, in part by US NIH grant U41 RR019703-028722 and US Army Medical Research and Materiel Command Grant W81XWH-08-1-0152.

\section{REFERENCES}

[1] P. R. Stepanishen and K. C. Benjamin, "Forward and backward projection of acoustic fields using FFT methods," $J$. Acoust. Soc. Am., 71, 803-812 (1982).

[2] R. Reibold and F. Holzer, "Complete mapping of ultrasonic fields without the wavelength limit," Acustica, 58, 11-16 (1985).

[3] H. Fleischer and V. Axelrad, "Restoring an acoustic source from pressure data using weiner filtering," Acustica, 60, 172175 (1986).

[4] M. Forbes, S. V. Letcher and P. R. Stepanishen, "A wave vector, time-domain method of forward projecting timedependent pressure fields," J. Acoust. Soc. Am., 90, 2782 2793 (1991).

[5] G. T. Clement, R. Liu, S. V. Letcher and P. R. Stepanishen, "Temporal backward planar projection of acoustic transients," J. Acoust. Soc. Am., 103, 1723-1726 (1998).

[6] O. A. Sapozhnikov, Y. A. Pishchal'nikov and A. V. Morozov, "Reconstruction of the normal velocity distribution on the surface of an ultrasonic transducer from the acoustic pressure measured on a reference surface," Acoust. Phys., 49, 354-360 (2003).

[7] T. L. Szabo, "Time-domain wave-equations for lossy media obeying a frequency power-law," J. Acoust. Soc. Am., 96, 491500 (1994).

[8] K. R. Waters, M. S. Hughes, J. Mobley, G. H. Brandenburger and J. G. Miller, "On the applicability of Kramers-Kronig relations for ultrasonic attenuation obeying a frequency power law," J. Acoust. Soc. Am., 108, 556-563 (2000).

[9] M. Liebler, S. Ginter, T. Dreyer and R. E. Riedlinger, "Full wave modeling of therapeutic ultrasound: Efficient timedomain implementation of the frequency power-law attenuation," J. Acoust. Soc. Am., 116, 2742-2750 (2004).

[10] G. V. Norton and J. C. Novarini, "Including dispersion and attenuation directly in the time domain for wave propagation in isotropic media," J. Acoust. Soc. Am., 113, 3024-3031 (2003).

[11] G. V. Norton and J. C. Novarini, "Finite-difference timedomain simulation of acoustic propagation in dispersive medium: An application to bubble clouds in the ocean," Comput. Phys. Commun., 174, 961-965 (2006).

[12] M. G. Wismer, "Finite element analysis of broadband acoustic pulses through inhomogenous media with power law attenuation," J. Acoust. Soc. Am., 120, 3493-3502 (2006).

[13] N. V. Sushilov and R. S. C. Cobbold, "Frequency-domain wave equation and its time-domain solutions in attenuating media,” J. Acoust. Soc. Am., 115, 1431-1436 (2004).

[14] J. D. Jackson, Classical Electrodynamics, 2nd ed. (John Wiley \& Sons, New York, 1975).

[15] L. Yadong and J. A. Zagzebski, "A frequency domain model for generating B-mode images with array transducers," IEEE Trans. Ultrason. Ferroelectr. Freq. Control, 46, 690-699 (1999).

[16] P. J. Westervelt, "Parametric acoustic array," J. Acoust. Soc. Am., 35, 535-537 (1963).

[17] D. T. Blackstock, "Transient solution for sound radiated into a viscous fluid," J. Acoust. Soc. Am., 41, 1312-1319 (1967).

[18] A. D. Pierce, Acoustics, An Introduction to Its Physical Principles and Applications (Acoustical Society of America, Woodbury, New York, 1989).

[19] G. T. Clement and K. Hynynen, "Forward planar projection through layered media," IEEE Trans. Ultrason. Ferroelectr. Freq. Control, 50, 1689-1698 (2003).

[20] G. T. Clement and K. Hynynen, "Field characterization of therapeutic ultrasound phased arrays through forward and backward planar projection," J. Acoust. Soc. Am., 108, 441446 (2000).

[21] M. Fink, "Time reversal of ultrasonic fields-Part I: Basic principles," IEEE Trans. Ultrason. Ferroelectr. Freq. Control, 39, 555-566 (1992).

[22] I. M. Hallaj, R. O. Cleveland, P. E. Barbone, S. G. Kargl and R. A. Roy, "Amplitude degradation of time-reversed pulses in nonlinear absorbing thermoviscous fluids," Ultrasonics, 38, 885-889 (2000).

[23] K. A. Wear, "A stratified model to predict dispersion in trabecular bone," IEEE Trans. Ultrason. Ferroelectr. Freq. Control, 48, 1079-1083 (2001).

[24] S. Chaffai, F. Padilla, G. Berger and P. Laugier, "In vitro measurement of the frequency-dependent attenuation in cancellous bone between 0.2 and $2 \mathrm{MHz}, " J$. Acoust. Soc. Am., 108(3 Pt 1), 1281-1289 (2000).

[25] F. J. Fry and J. E. Barger, "Acoustic properties of the human skull,” J. Acoust. Soc. Am., 63, 1576-1590 (1978).

[26] G. T. Clement and K. Hynynen, "Correlation of ultrasound phase with physical skull properties," Ultrasound Med. Biol., 28, 617-624 (2002). 